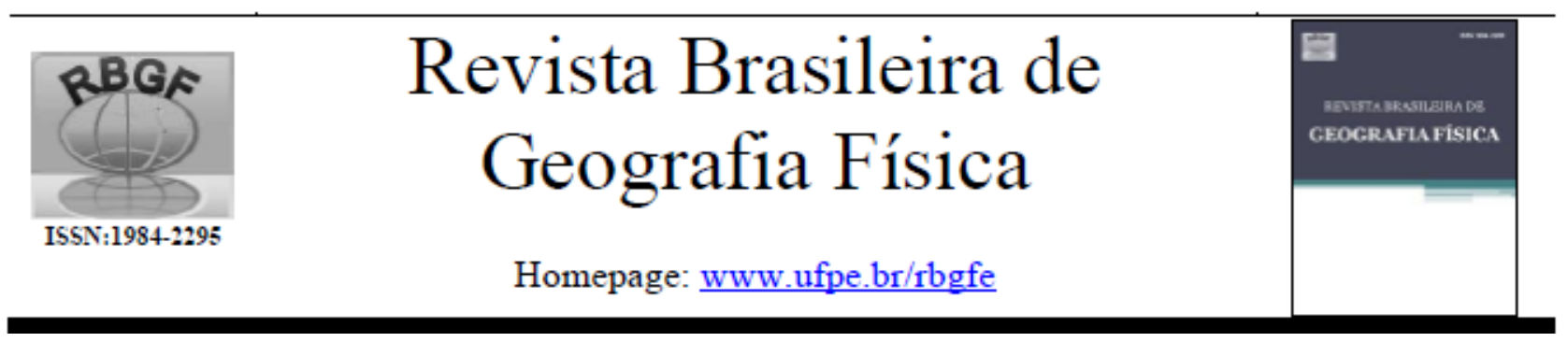

\title{
Estudo climático dos eventos de precipitação associados a alagamentos urbanos no Estado do Rio Grande do Sul
}

\author{
Rodrigo da Silva Pereira ${ }^{1}$; André Becker Nunes².
}

${ }^{1}$ Mestrando em Meteorologia no PPGMET/UFPEL, e-mail: psdrigo@gmail.com (autor correspondente). Endereço: Av. Ildefonso Simões Lopes, 2751, Bairro Arco-Íris, Pelotas-RS, 96060-290; ${ }^{2}$ Professor do PPGMET/UFPEL, andre.nunes@ufpel.edu.br, Endereço: Av. Ildefonso Simões Lopes, 2751, Bairro Arco-Íris, Pelotas-RS, 96060-290

Artigo recebido em 12/09/2018 e aceito em 20/12/2018

\section{R E S U M O}

Eventos de chuva intensa são caracterizados por altas taxas de precipitação que acarretam em vários transtornos sociais. Contudo, uma determinada taxa de precipitação que provoca transtornos em uma cidade pode não representar riscos em outra, dado que cada local tem sua própria geografia. Este trabalho, baseado no histórico de alagamentos, tem como objetivo a análise dos eventos de precipitação separando-os em casos de atenção (menos severos) e alerta (mais severos) para os quatro maiores centros urbanos do Rio Grande do Sul: Porto Alegre, Pelotas, Caxias do Sul e Santa Maria. Porto Alegre, a cidade mais urbanizada, é a que apresenta menores limiares, indicando maior facilidade para a ocorrência destes eventos. Os limiares foram aplicados nos dados observados para a análise das ocorrências do período de 1961-2013. Santa Maria apresentou o número maior de casos, e Porto Alegre apresentou uma maior proporção de casos de alerta sobre os casos totais. Os resultados mostram que, no geral, há muita variabilidade anual no número de casos, com leve tendência positiva, sendo o inverno a estação preponderante. Além disso, verificou-se, na maior parte, correlação moderada entre o número de casos e as fases do El Niño e do Índice do Atlântico Sul.

Palavras-chave: chuvas; eventos extremos; El Niño; variabilidade climática; alerta

\section{Climatic study of rainfall events related to urban flash floods in Rio Grande do Sul State}

\section{A B S T R A C T}

Intense precipitation events are characterized by high rainfall rates that lead to several social problems. However, a specific rainfall rate that causes damage in one city may not mean risks in another, since each location has its own geographical characteristics. Based on flooding records, the aim of this work is to analyze the precipitation events separating them in attention cases (less severe) and warning cases (more severe) for the four largest urban centers of Rio Grande do Sul: Porto Alegre, Pelotas, Caxias do Sul and Santa Maria. Porto Alegre, the most urbanized city, is the one with the lowest thresholds, indicating greater chance for the occurrence of such events. Thresholds were applied on observed dataset in order to analyze the occurrences on 1961-2013 period. Santa Maria presented the highest number of cases, and Porto Alegre presented the higher proportion of warning cases on total cases. Overall, the results show great annual variability of number of cases, with slight positive trend, being winter the preponderant season. In addition, there was generally a moderate correlation between number of cases and El Niño and South Atlantic Index phases.

Keywords: rainfall; extreme events; El Niño; climatic variability; warning

\section{Introdução}

A urbanização das cidades acarreta, normalmente, em mudanças na cobertura natural da superfície, propiciando menos absorção da precipitação e aumento do escoamento superficial que, por sua vez, provoca o alagamento. Castro (2003) define alagamento como "águas acumuladas no leito de ruas e nos perímetros urbanos por fortes precipitações pluviométricas em cidades com sistemas de drenagens deficientes" definição similar é encontrada no National Severe Storms Laboratory, disponível em http://www.nssl.noaa.gov/education/svrwx101/flo ods/types/. Ou seja, o alagamento é um transtorno tipicamente urbano, sendo mais frequente quanto mais propício à eventos de chuva intensa for o 
local. Uma vez que um evento de chuva intensa é categorizado como evento raro (localizado nos extremos em uma distribuição de frequência) e que provoca algum tipo de transtorno, podemos chamálo de evento extremo de precipitação. Tais eventos podem ter como consequência desde problemas no trânsito até deslizamentos de terra. Por este motivo o tema sempre exigiu muita atenção da comunidade científica especializada.

Um evento extremo de precipitação é identificado a partir de uma determinada taxa, ou limiar $\left(\mathrm{mm} \mathrm{dia}^{-1}\right)$, obtida de acordo com alguma metodologia. $\mathrm{Na}$ literatura existem muitas metodologias para a identificação de um evento extremo de precipitação, como as encontradas em Houze et al. (1990), Karl et al. (1995), Groisman et al. (1999), Harnack et al. (1999), Haylock e Nicholls (2000), Haylock et al. (2006), Teixeira e Satyamurty (2007), entre outros. No Brasil existe bom número de estudos que identificam eventos extremos de precipitação baseados em diferentes metodologias, para diferentes locais, como por exemplo Carvalho et al. (2002), seguindo metodologia de Liebmann et al. (2001), Oliveira (2011), Andrade e Pinheiro (2011), Camargo et al. (2011), Nunes e Da Silva (2013), Loureiro et al. (2014) e Campos et al. (2015). Uma vez compreendido que os alagamentos são fenômenos urbanos, torna-se necessário que cada cidade tenha seu próprio limiar para a detecção de evento extremo de precipitação. Afinal, um determinado limiar de uma cidade não necessariamente implica na ocorrência de um evento extremo em outra, haja vista que cada cidade tem sua própria geografia natural (relevo e cobertura da superfície) e urbanização (ocupação de área verde, mudança da cobertura do solo), exigindo estudos específicos para cada local (Da Silva e Nunes, 2011; Nunes e Da Silva, 2013).

Haja vista que a urbanização exerce influência na ocorrência de alagamentos urbanos, estes, por sua vez, tendem a serem mais frequentes devido ao contínuo crescimento das cidades brasileiras. Somado a este fato, conforme Carvalho et al. (2014), por exemplo, existe uma tendência positiva nos máximos anuais de precipitação diária nas regiões sul, sudeste e centro-oeste do Brasil.

O Estado do Rio Grande do Sul (RS), além de se localizar em uma região favorável à ocorrência de eventos de precipitação intensa (Grimm, 2009), possui boa parte de seu território com relevo acidentado, o que propicia mais casos de alagamentos urbanos. Quanto a variabilidade climática, se por um lado o RS não apresenta grandes variações sazonais dos valores de precipitação, por outro é influenciado pelo fenômeno ENOS (El Niño-Oscilação Sul) e pelas variações de TSM (temperatura da superfície do mar) do Atlântico Sul, como observado, por exemplo, em Santos e Diniz (2014).

Este trabalho tem como objetivo a análise climática dos eventos de precipitação local que tem potencial para causar alagamentos nos quatro principais centros urbanos do Rio Grande do Sul: Porto Alegre, Caxias do Sul, Pelotas e Santa Maria. Por se tratar de alagamentos urbanos, não serão considerados aqui casos de enchentes devido a cheias de rios - o que no caso dos centros em questão são raros. A análise consiste de três etapas: 1) obtenção dos limiares de cada cidade para identificação dos casos com potencial para alagamentos; 2) quantificação do número de casos e obtenção das tendências e 3) correlações entre as ocorrências e algumas das oscilações climáticas que mais influenciam na variabilidade climática da região.

\section{Material e métodos}

O Estado do Rio Grande do Sul está localizado na região mais meridional do Brasil, aproximadamente entre as latitudes de $30^{\circ} \mathrm{S}$ e $34^{\circ} \mathrm{S}$, o que o torna suscetível aos sistemas meteorológicos subtropicais à oeste e aos sistemas extratropicais, como ondas baroclínicas, à oeste e sul (Satyamurty et al., 1998). Frentes frias e sistemas convectivos de mesoescala são os sistemas de precipitação predominantes na região, ocorrendo o ano todo. As frentes frias são mais importantes no inverno, enquanto que os sistemas convectivos são mais preponderantes nas estações mais quentes (Grimm et al., 1998; Grimm, 2009). Eventualmente a região é atingida por ciclones extratropicais formados à sotavento dos Andes, na Planície do Chaco (região norte da Argentina), que se movem para sudeste em direção ao Oceano Atlântico (Gan e Rao, 1991; Gan e Seluchi, 2009) especialmente devido às forçantes quasegeostróficas advectivas observadas em diferentes níveis verticais (Nunes, 2017). Sistemas precipitantes provenientes do Atlântico são menos comuns (Grimm, 2009).

Neste estudo foram analisadas as quatro cidades mais populosas do RS: a capital Porto Alegre (POA), Pelotas (PEL), Caxias do Sul (CXS) e Santa Maria (SM) (Figura 1). Além de serem as cidades mais urbanizadas do Estado (desconsiderando aqui a cidade de Canoas, por ser satélite da capital), essas cidades são relativamente distantes entre si, representando polos regionais e, geograficamente, possuem diferentes relevos. De acordo com o Instituto Brasileiro de Geografia e Estatística (IBGE), Porto Alegre $\left(30^{\circ} 02^{\prime} \mathrm{S}\right.$, $\left.51^{\circ} 14^{\prime} \mathrm{O}\right)$, na região nordeste do Estado, possui 1,4 
milhões de habitantes, tem geografia física diversificada, com poucos morros e várzeas e uma altitude média de $10 \mathrm{~m}$. Pelotas ( $\left(31^{\circ} 46^{\prime} \mathrm{S}, 52^{\circ} 21^{\prime} \mathrm{O}\right)$ está localizada na região sul do Estado, com uma população de $328 \mathrm{mil}$ habitantes e apresenta uma orografia plana com altitude média de $7 \mathrm{~m}$. Caxias do $\operatorname{Sul}\left(29^{\circ} 10^{\prime} \mathrm{S}, 51^{\circ} 11^{\prime} \mathrm{O}\right)$, com 440 mil habitantes, está localizada na região nordeste do Estado, conhecida como Serra Gaúcha devido ao relevo acidentado e altitude média de $817 \mathrm{~m}$. Santa Maria $\left(29^{\circ} 41^{\prime} \mathrm{S}, 53^{\circ} 48^{\prime} \mathrm{O}\right)$ localiza-se na região central do Estado. Possui 262 mil habitantes, tem terreno acidentado e altitude média de $151 \mathrm{~m}$.

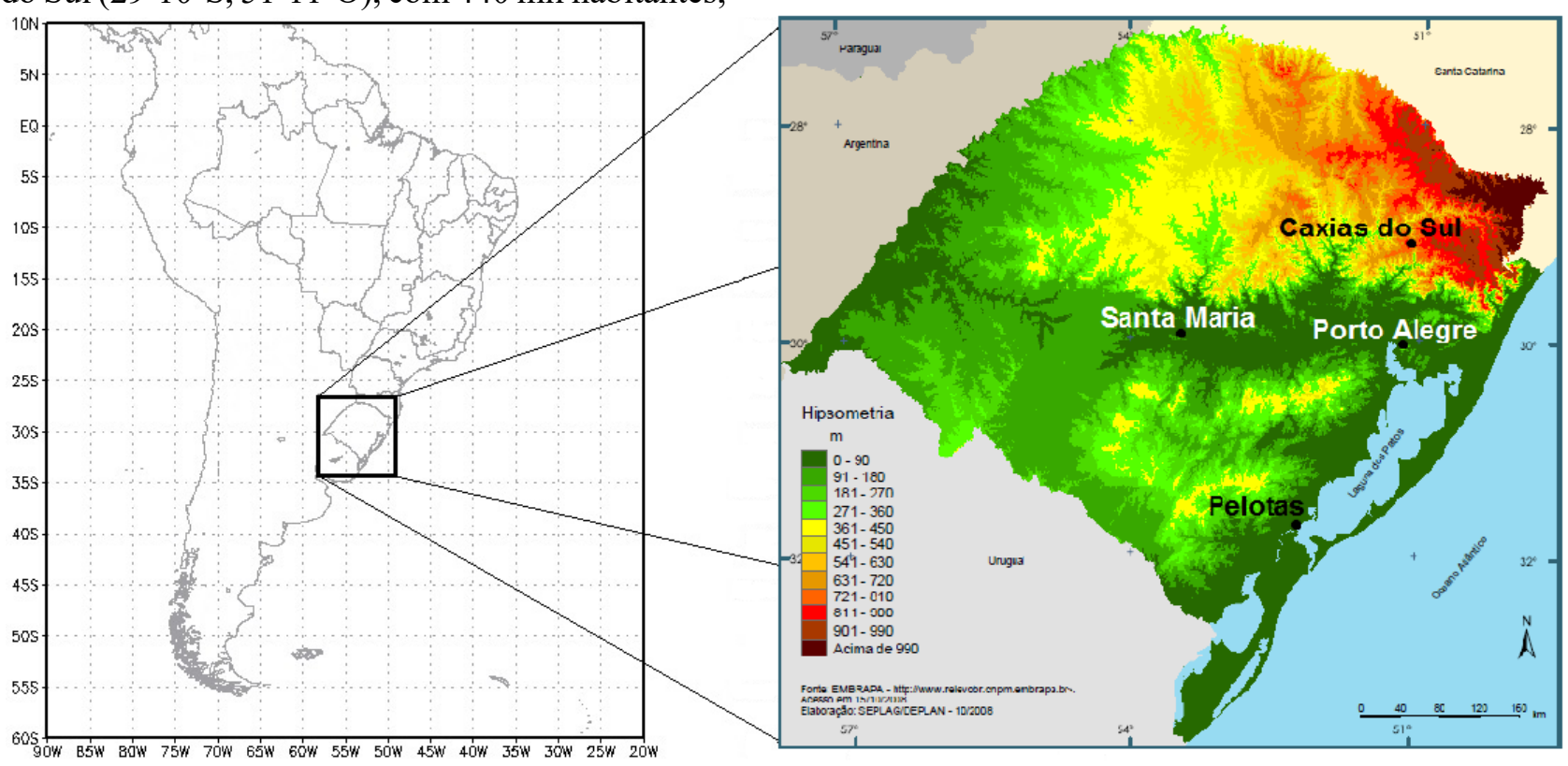

Figura 1 Topografia (m) do Estado do Rio Grande do Sul (ampliado à direita) com a localização das cidades selecionadas.

Para que de alguma forma sejam levadas em consideração as características peculiares de cada cidade, foram observadas as precipitações acumuladas nas 48 horas anteriores a cada evento de alagamento urbano. Tais valores de precipitação foram organizados e os percentis obtidos para a identificação dos limiares para a detecção dos eventos extremos de precipitação com potencial para causar alagamentos. Foram observados os casos de alagamentos do período de 2003 à 2013. As principais fontes de informação sobre os casos de alagamento foram a Defesa Civil do Estado do Rio Grande do Sul e jornais locais. Tais informações foram confrontadas com os dados de precipitação observada para que se filtrasse qualquer erro, como os erros na informação da data e local exatos do alagamento.

Os dados de precipitação diária foram obtidos, basicamente, das estações meteorológicas do Instituto Nacional de Meteorologia (INMET) de cada uma das cidades. Considerou-se o limiar de $2 \%$ de falhas para se considerar o ano de dados como válido. A estação de Pelotas não apresentou nenhuma falha nos dados de precipitação, Porto Alegre apresentou menos de $2 \%$, Caxias apresentou pouco mais de $2 \%$, e os dados com falhas (ou ausentes) foram substituídos pelos dados da estação da Agência Nacional de Águas (ANA).
A estação de Santa Maria apresentou falhas bem maiores que este limiar nos anos de 1967 e 1968, e como tais falhas não puderam ser substituídas por dados da estação da ANA este período não pode ser considerado neste estudo. Salienta-se que as falhas aqui citadas tem caráter praticamente homogêneo quanto à estação do ano, de modo que a análise sazonal não ficou prejudicada. Dados dessas fontes têm sido empregados em diversos trabalhos, para diferentes fins, como por exemplo, Grimm (2003), Teixeira e Satyamurty (2011) e Blunden e Arndt (2012).

Dois tipos de eventos foram considerados de acordo com a intensidade da precipitação acumulada em 48 horas associada aos casos de alagamento: casos de atenção, com limiares de acordo com o percentil 25, e casos de alerta, de acordo com o percentil 75 dos valores de precipitação. Essa separação dos casos em dois tipos torna-se necessária devido às consequências provocadas pelos mesmos, haja vista que casos de atenção tendem a provocar pequenos danos, como transtornos de trânsito, enquanto que casos de alerta podem provocar consequências mais sérias, como desalojamento de pessoas ou deslizamentos de terra. Uma vez obtidos os limiares, os mesmos foram aplicados nos dados de precipitação observada no período de 1964-2013 (50 anos, 
exceto em Santa Maria onde são 48 anos) para que se identifiquem os possíveis casos de atenção e alerta durante o período.

A possível influência das oscilações climáticas nas ocorrências dos casos de atenção e alerta foi analisada por meio de correlação linear entre índices climáticos e o acumulado mensal de ocorrências. Para a análise da influência do ENOS canônico foi empregado o índice IME (Índice Multivariado do El Niño) (Wolter, 1987), e para a análise do El Niño Modoki, o Índice Modoki (Ashok et al., 2007). A influência das anomalias de TSM do Atlântico Sul foi analisada de acordo com os dados obtidos por Santos e Diniz (2014). A correlação linear foi considerada estatisticamente significativa, de acordo com o teste T-Student (Wilks, 2011), para valores de confiança igual ou maiores que $95 \%$.

\section{Resultados e discussão}

A Tabela 1 apresenta os limiares para os casos de atenção e alerta de 48 horas de precipitação acumulada. $\mathrm{O}$ fato de Porto Alegre apresentar limiares menores era esperado, haja vista que é a cidade mais urbanizada, com maior área de cobertura superficial não-natural, o que aumenta o escoamento superficial e, consequentemente, a chance de alagamento. Ou seja, na capital é necessária uma taxa menor de precipitação para a ocorrência de alagamentos.

Tabela 1 Limiares (mm) de precipitação acumulada em 48 horas para detecção dos casos de atenção e alerta.

\begin{tabular}{c|c|c}
\hline Cidade & Atenção & Alerta \\
\hline Porto Alegre & 25,2 & 53,5 \\
\hline Pelotas & 36,4 & 80,5 \\
\hline Caxias do Sul & 33,2 & 84,0 \\
\hline Santa Maria & 30,5 & 76,9 \\
\hline
\end{tabular}

Com estes limiares os casos de atenção e alerta foram somados em quinquênios, de 1964 à 2013 (Figuras 2-5), o que gera uma tendência linear mais clara do que a anual.

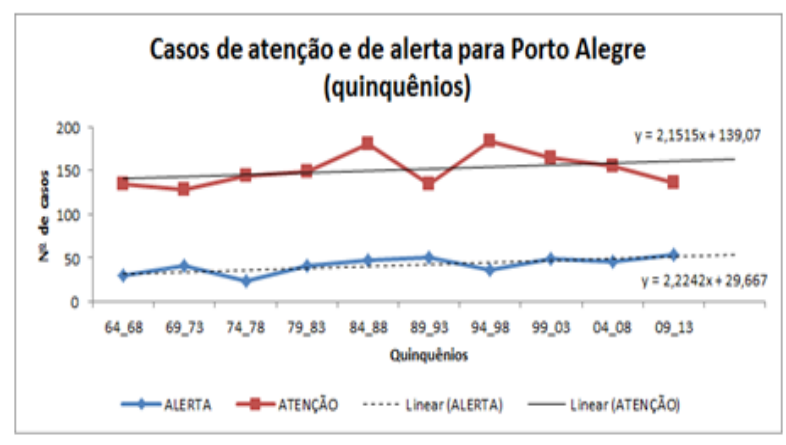

Figura 2 Eventos com potencial de alagamentos em Porto Alegre.

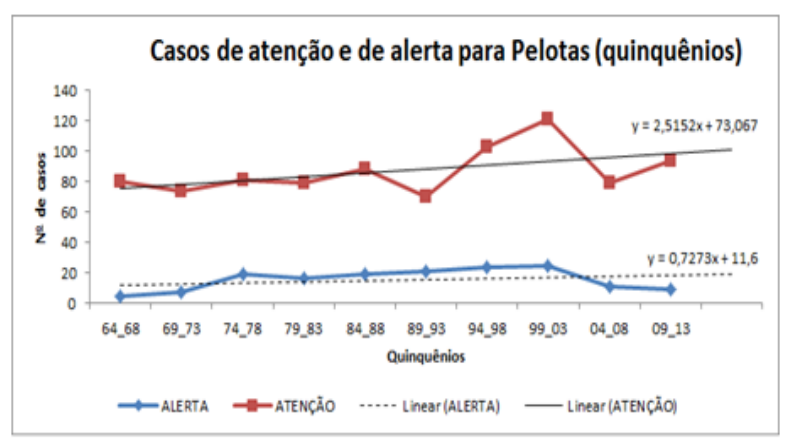

Figura 3 Eventos com potencial de alagamentos em Pelotas

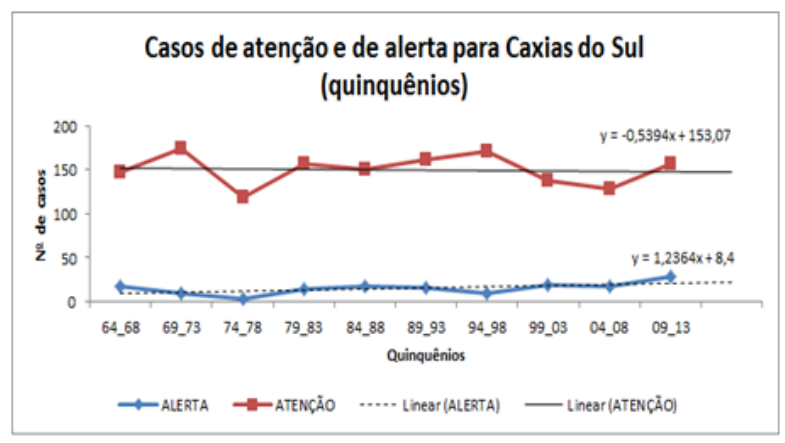

Figura 4 Eventos com potencial de alagamento em Caxias do Sul

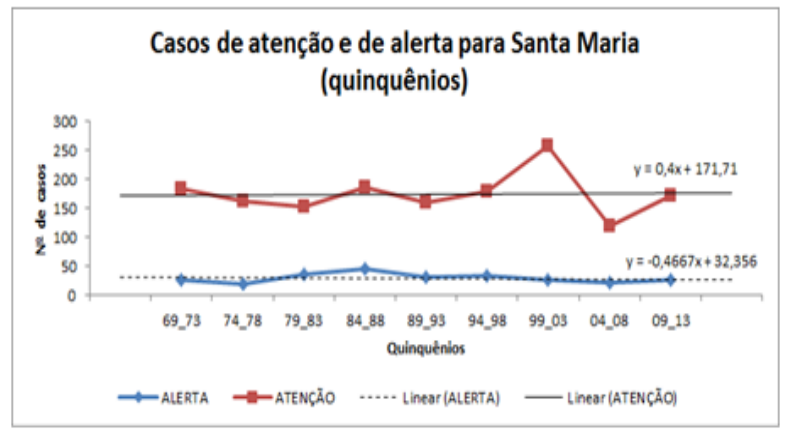

Figura 5 Eventos com potencial de alagamento em Santa Maria

Independente do tipo de evento, se alerta ou atenção, observa-se tendências lineares positivas, embora muito sutis, para todas as cidades, exceto com relação aos casos de alerta em Santa Maria e atenção em Caxias do Sul, onde se observa 
tendências levemente negativas. Ou seja, das oito situações aqui estimadas, em seis se observa um aumento no número de eventos de chuva intensa, o que concorda com os trabalhos que indicam aumento de eventos extremos no globo (Diffenbaugh et al., 2018). Pelotas apresenta o maior coeficiente linear para os casos de atenção, enquanto Porto Alegre apresenta o maior coeficiente para os casos de alerta.

A análise sazonal (Figuras 6 a 9 ) mostra predominância do inverno, aqui considerado como Julho-Agosto-Setembro (JAS), para os casos de atenção. Tal comportamento se observa com mais clareza na capital. O inverno também é a estação preponderante para os casos de alerta, exceto em S. Maria, onde se observa pouca variação entre as estações do ano. Desta forma, ressalta-se, portanto, o papel dos sistemas frontais, mais frequentes e intensos no inverno, nas ocorrências de eventos de precipitação intensa.

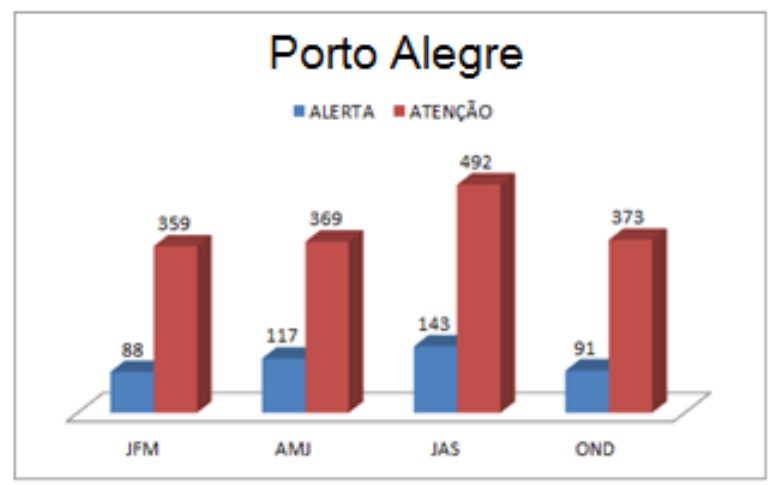

Figura 6 Casos de atenção e alerta (1964-2013) em Porto Alegre para cada estação do ano, onde JFM é Janeiro-Fevereiro-Março, AMJ é Abril-MaioJunho, JAS é Julho-Agosto-Setembro e OND é Outubro-Novembro-Dezembro..

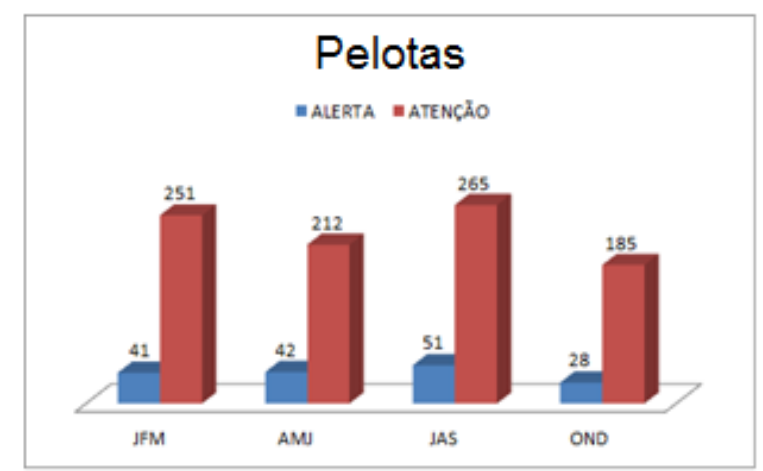

Figura 7 Casos de atenção e alerta (1964-2013) para Pelotas para cada estação do ano.

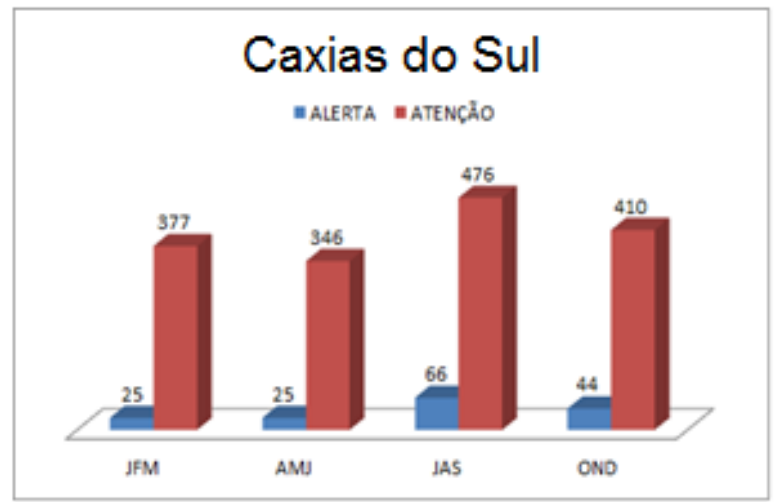

Figura 8 Casos de atenção e alerta (1964-2013) para Caxias do Sul para cada estação do ano.

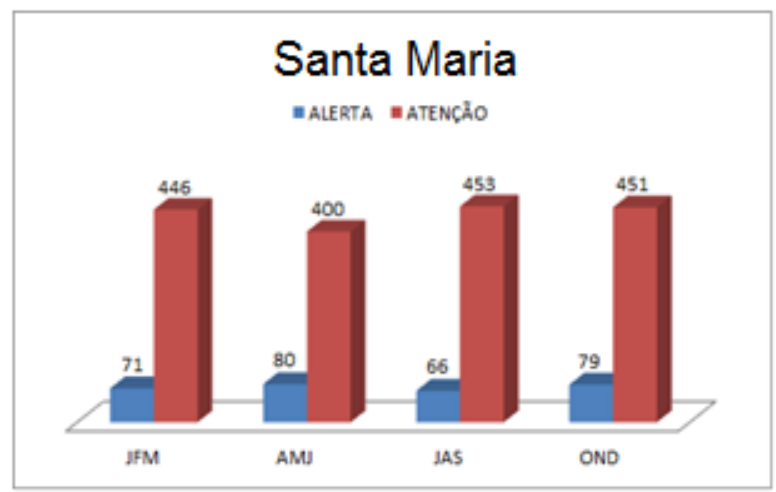

Figura 9 Casos de atenção e alerta (1964-2013) para Santa Maria para cada estação do ano.

A seguir, é apresentada a relação entre as ocorrências dos eventos de precipitação intensa e o índice IME, para que seja observada a existência de associação entre tais eventos e o fenômeno ENOS. As figuras 10 à 13 mostram que, visualmente, existe alguma relação entre as variáveis, especialmente quanto aos casos de alerta. Para Porto Alegre (Figura 10) esta relação entre os casos de alerta e o IME apresenta um coeficiente de correlação linear de 0.33 para uma significância estatística de $99.3 \%$. Já o coeficiente de correlação entre os casos de atenção e o IME ficou em 0.26 para uma significância estatística de $97 \%$. Com tais valores de coeficiente de correlação linear, pode-se considerar, conforme Dancey e Reidy (2006) que as relações entre os casos com potencial para causar alagamentos em POA e o índice IME são fracas.

Para a cidade de Pelotas (Figura 11), a relação entre os casos de atenção e o índice IME apresentou coeficiente de correlação linear de 0.34 (fraco) para uma significância estatística de $99.2 \%$, enquanto que a relação entre os casos de alerta e o IME ficou em 0.43 (moderada) para uma significância de $99.9 \%$.

Para Caxias do Sul (Figura 12) o coeficiente de correlação linear entre os casos de atenção e o IME ficou em 0.37 , com significância estatística de 
99.6\%. Já para os casos de alerta, o coeficiente de correlação ficou em 0.11 e não atingiu a significância estatística mínima de 95\%.

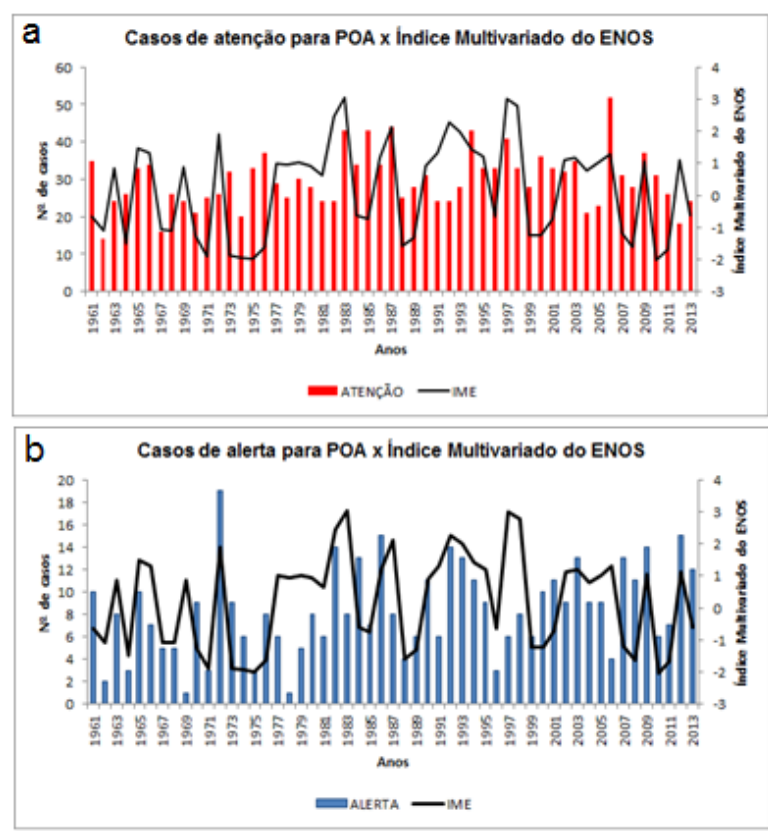

Figura 10 Variação anual das ocorrências (barras) e o índice IME (linhas) para Porto Alegre. Casos de atenção (a) e alerta (b).

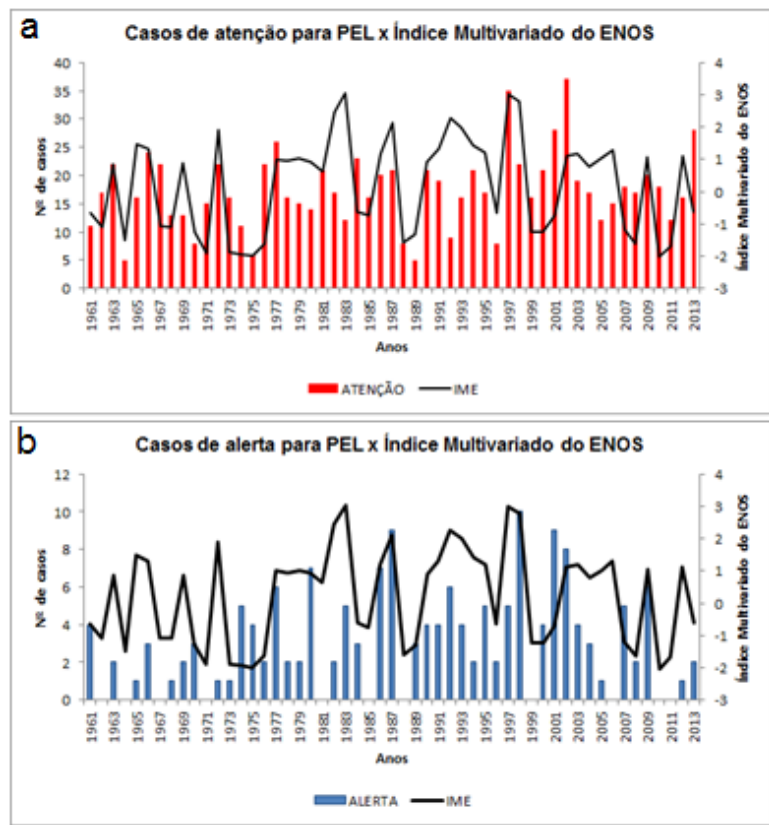

Figura 11Variação anual das ocorrências (barras) e o índice IME (linhas) para Pelotas. Casos de atenção (a) e alerta (b).

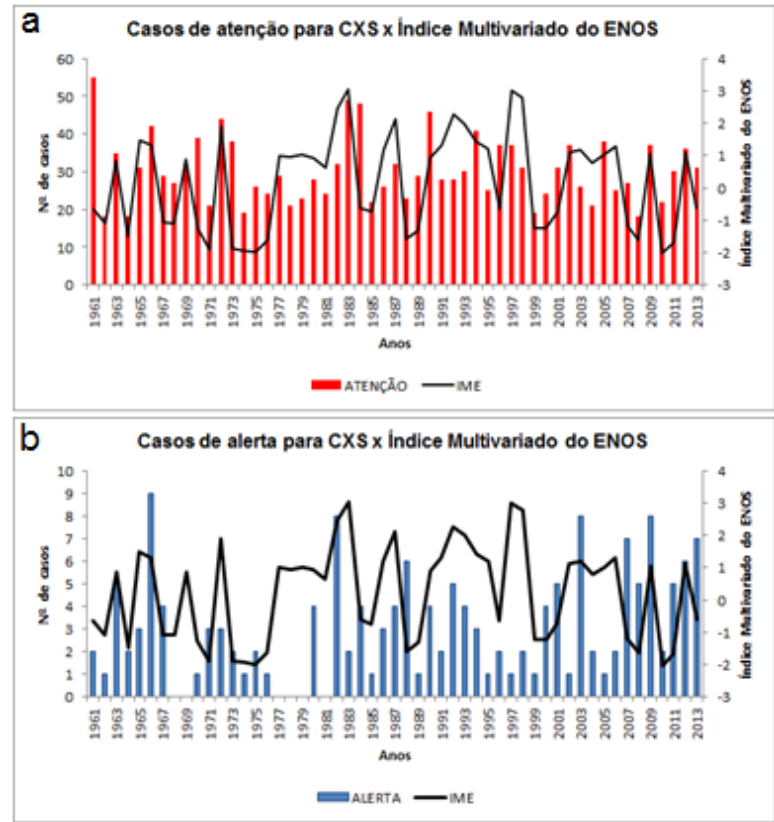

Figura 12 Variação anual das ocorrências (barras) e o índice IME (linhas) para Caxias do Sul. Casos de atenção (a) e alerta (b).

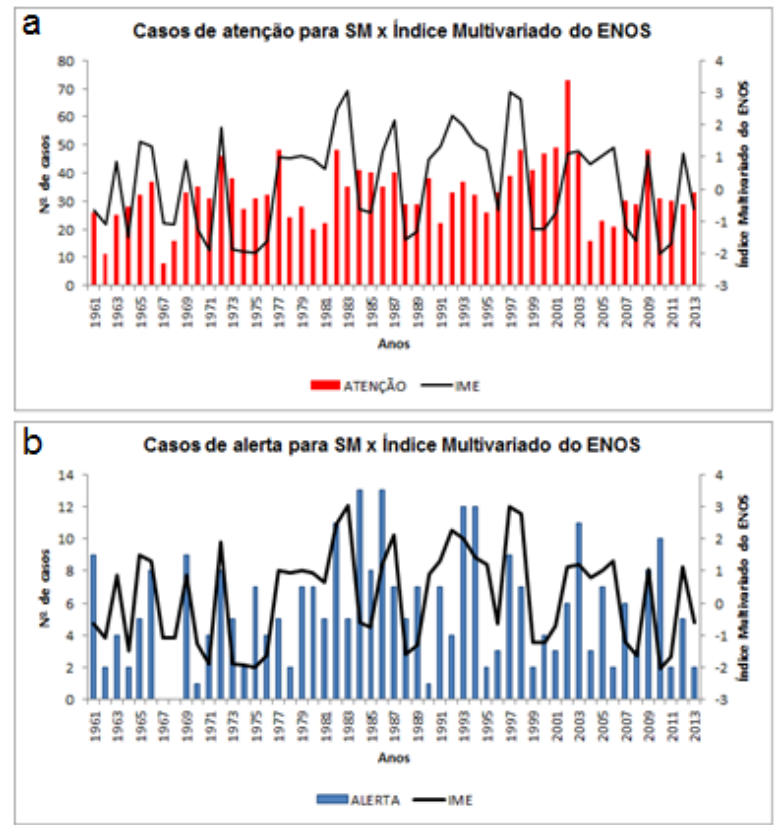

Figura 13 Variação anual das ocorrências (barras) e o índice IME (linhas) para Santa Maria. Casos de atenção (a) e alerta (b).

Para Santa Maria, o coeficiente entre os casos de atenção e IME ficou em 0.25 (fraco), para 96.2\% de significância, e entre os casos de alerta e IME, 0.38 (moderado) para significância de $99.7 \%$. Ou seja, dado que as correlações são todas positivas, existe uma maior chance de ocorrência dos eventos de chuva intensa em épocas de El Niño do que em épocas de La Niña.

Por se tratar de casos de eventos de precipitação associados a casos de alagamentos, não se esperava aqui grandes valores de coeficiente 
de correlação entre as ocorrências e os índices climáticos, afinal, tais eventos são influenciados por diversos aspectos da geografia local. Mesmo assim, os resultados concordam em parte com demais estudos de climatologia (Tedeschi et al., 2015, por exemplo) que indicam conexão do fenômeno El Niño com eventos severos de precipitação no sul do Brasil.

A relação entre as ocorrências de casos de atenção e alerta e o índice IAS (gráficos não mostrados aqui) mostrou-se ligeiramente inferior à relação com o índice MEI. Destaca-se, contudo, a relação entre os casos de alerta em Pelotas (coeficiente de correlação linear de 0.39 , com 99.9\% de significância estatística), de atenção em Caxias do Sul (0.29, com significância de $98 \%)$ e de atenção em Santa Maria $(0.43$, com significância de $99.7 \%$ ). Ou seja, nestas situações, existe relação entre o aquecimento do oceano Atlântico adjacente e determinadas ocorrências de eventos de chuva.

Com relação ao índice Modoki (gráficos não mostrados aqui), não se encontrou nenhuma situação com significância estatística, independente do tipo de caso ou cidade.

\section{Conclusões}

No presente trabalho sugeriu-se limiares para a deteç̧ão de eventos de precipitação com potencial para alagamentos urbanos nas cidades de Porto Alegre, Pelotas, Caxias do Sul e Santa Maria empregando o histórico de alagamentos urbanos. Os limiares indicam que em Porto Alegre tais eventos, sejam casos de alerta (mais grave) ou atenção, ocorrem com menores taxas de precipitação.

Durante o período de 1963-2013, observou-se, no geral, que ambos casos apresentaram tendências lineares positivas, especialmente em Porto Alegre e Pelotas, porém muito sutis.

Também na capital se observa, com mais clareza, que o inverno é a estação preponderante tanto para casos de atenção quanto de alerta, exceto em Santa Maria onde não se observa variações sazonais relevantes.

Apesar de, no geral, serem encontrados valores baixos de correlação linear, nota-se uma maior chance de ocorrência de eventos em períodos de El Niño, de acordo com o índice MEI, e em períodos de anomalias positivas da TSM do Atlântico Sul, de acordo com o índice IAS. Não se encontrou relação significativa entre as ocorrências dos eventos e o El Niño Modoki.
O primeiro autor agradece à CAPES pela bolsa de mestrado.

\section{Referências}

Andrade, K.M.; Pinheiro, H.R. 2011. Simulação de eventos extremos de precipitação na região serrana do Rio de Janeiro no clima presente e futuro utilizando o modelo Eta-HadCM3. IV Simpósio Internacional de Climatologia. 16 a 19 de outubro.

Ashok, K.; Behera, S.K.; Rao, S. A.; Weng, H.; Yamagata, T. 2007. El Niño Modoki and its possible teleconnection. Journal of Geophysical Research 112, C11007.

Blunden, J.; Arndt, D.S. 2012. State of the climate in 2011. Bulletin of the American Meteorological Society 93, S1-S282.

Camargo, C.G.; Braga, H.; Malandrin, D.A.; Machado, L. 2011. Análises de eventos extremos de precipitação na Região Sul do Brasil: Dados históricos. XVII Congresso Brasileiro de Agrometeorologia. 18 a 21 de julho.

Campos, T.L.O.B.; Mota, M.A.S.; Santos, S.R.Q. 2015. Eventos extremos de precipitação em Belém-PA: uma revisão de notícias históricas de jornais. Revista Ambiente \& Água 10, 182194.

Carvalho, J.R.P.; Assad, E.D.; Oliveira, A.F.; Pinto, H.S. 2014. Annual maximum daily rainfall trends in the Midwest, Southeast and Southern Brazil in the last 71 years. Weather and Climate Extremes 5-6, 7-15.

Carvalho, L.M.V.; Jones, C.; Liebmann, B. 2002. Extreme precipitation events in Southeatern South America and large-scale convective patterns in the South Atlantic Convergence Zone. Journal of Climate 15, 2377-2394.

Castro, A.L.C. 2003. Manual de Desastres: Desastres Naturais, Vol. I. Ministério da Integração Nacional - Secretaria Nacional de Defesa Civil, Brasília.

Da Silva, G.C.; Nunes, A.B. 2011. Análise de eventos extremos de precipitação no leste de Santa Catarina: Estudo de tendência. Ciência e Natura 33, p. 251-254. DOI 10.5902/2179460X9432

Dancey, C. P.; Reidy, J. 2006, Estatística Sem Matemática para Psicologia: Usando SPSS para Windows, 3ed. Artmed, Porto Alegre.

Diffenbaugh, N.S.; Singh, D.; Mankin, J.S. 2018. Unprecedented climate events: Historical changes, aspirational targets, and national commitments. Science Advances 4. DOI 10.1126/sciadv.aao3354

\section{Agradecimentos}


Gan, M.A.; Rao, V.B. 1991. Surface cyclogenesis over South America. Monthly Weather Review 119, 1293-1303.

Gan, M.A.; Seluchi, M.E. 2009. Ciclones e Ciclogênese. In: Cavalcanti, I.F.A.; Ferreira, N.J.; Justi da Silva, M.G.A.; Silva Dias, M.A.F. (Organizadores), Tempo e Clima no Brasil. Oficina de Textos, São Paulo.

Grimm, A.M.; Ferraz, S.E.T.; Gomes, J. 1998. Precipitation anomalies in Southern Brazil associated with El Niño and La Niña events. Journal of Climate 11, 2863-2880.

Grimm, A.M. 2003. The El Niño impact on the summer monsoon in Brazil: Regional processes versus remote influences. Journal of Climate 16, 263-280.

Grimm, A.M. 2009. Clima da Região Sul do Brasil. In: Cavalcanti, I.F.A.; Ferreira, N.J.; Justi da Silva, M.G.A.; Silva Dias, M.A.F. (Organizadores), Tempo e Clima no Brasil. Oficina de Textos, São Paulo.

Groisman, P.Y.; Karl, T.R.; Easterling, D.R.; Knight, R.W.; Jameson, P.F.; Hennessy, K.J.; Suppiah, R.; Page, C.M.; Wibig, J.; Fortuniak, K.; Razuvaev, V.N.; Douglas, A.; Forland, E.; Zhai, P-M. 1999. Changes in the probability of heavy precipitation: important indicators of climatic change. Climatic Change 42, 243-283.

Harnack, R.P.; Apffel, K.; Cermack, J.R. 1999. Heavy precipitation events in New Jersey: attendant upper air conditions. Weather Forecasting 14, 933-954.

Haylock, M.R.; Nicholls, N. 2000. Trends in extreme rainfall indices for an updated high quality data set for Australia, 1910-1998. International Journal of Climatology 20, 15331541.

Haylock, M.R.; Peterson, T.C.; Alves, L.M.; Ambrizzi, $\quad$ T.; Anunciação, Y.M.T.; Baez, J.; Barros, V.R.; Berlato, M.A.; Bidegain, M.; Coronel, G.; CORRADI, V.; Garcia, V.J.; Grimm, A.M.; Karoly, D.; Marengo, J.A.; Marino, M.B.; Moncunill, D.F.; Nechet, D.; Quintana, J.; Rebello, E.; Rusticucci, M.; Santos, J.L.; Trebejo, I. ; Vincent, L.A. 2006. Trends in Total and Extreme South American Rainfall in 19602000 and Links with Sea Surface Temperature. Journal of Climate 19, 1490-1512.

Houze, R.A.; Smull, B.F.; Dodge, P. 1990. Mesoscale organization of springtime rainstorms in Oklahoma. Monthly Weather Review 118, 613-654.

Karl, T.R.; Knight, R.W.; Easterling, D.R.; Quayle, R.G. 1995. Trends in U.S. climate during the twentieth century. Consequences 1, 2-12.
Liebmann, B.; Jones, C.; Carvalho, L.M.V. 2001. Interannual variability of daily extreme precipitation events in the State of São Paulo, Brazil. Journal of Climate 14, 208-218.

Loureiro, R.S.; Saraiva, J.M.; Saraiva, I.; Senna, R.C.; Fredó, A.S. 2014. Estudo dos eventos extremos de precipitação ocorridos em 2009 no Estado do Pará. Revista Brasileira de Meteorologia 29, 83-94.

Nunes, A.B. 2017. Case Study of Upper Tropospheric Meteorological Systems on South America: Synoptic Analysis. Anuário do Instituto de Geociências - UFRJ 40, 83-92. DOI: 10.11137/2017_1_70_82

Nunes, A.B.; Da Silva, G.C. 2013. Climatology of extreme rainfall events in eastern and northern Santa Catarina State, Brazil: present and future climate. Revista Brasileira de Geofísica 31, 413-425. DOI: 10.22564/rbgf.v31i3.314

Oliveira, N. 2011. Análise da evolução de eventos extremos de precipitação diária na cidade de São Paulo. IV Simpósio Internacional de Climatologia. 16 a 19 de outubro.

Santos, E. B.; Diniz, G.B. 2014. Oceanic indices and their relations with the monthly precipitation in Rio Grande do Sul State, Brazil. Revista Brasileira de Geofísica 32, 371-381.

Satyamurty, P.; Nobre, C.A.; Silva Dias, P.L. 1998. Meteorology of the Tropics: South America. In: Karoly, D. J.; Vincent, D. G. (editores). Meteorology of Southern Hemisphere. Meteorological Monographs, v. 27, n. 49, chapter 3A.

Tedeschi, R.G.; Grimm, A.M.; Cavalcanti, I.F.A. 2015. Influence of central and east ENSO on extreme events of precipitation in South America during austral spring and summer. International Journal of Climatology 30, 20452064.

Teixeira, M.S.; Satyamurty, P. 2007. Dynamical and synoptic characteristics of heavy rainfall episodes in Southern Brazil. Monthly Weather Review 135, 598-617.

Teixeira, M.S.; Satyamurty, P 2011. Trends in the frequency of intense precipitation events in Southern and Southeastern Brazil during 19602004. Journal of Climate 24, 1913-1921.

Wilks, D. S. 2011. Statistical Methods in the Atmospheric Sciences. Academic Press, Burlington.

Wolter, T. 1987. The Southern Oscillation in surface circulation and climate over the Tropical Atlantic, Eastern Pacific, and Indian Oceans as captured by cluster analysis. Journal of Climate and Applied Meteorology 26, 540558. 